\title{
Co-effect of insulin resistance and biomarkers of inflammation and endothelial dysfunction on hypertension
}

\author{
Hongmei $\mathrm{Li}^{1}$, Xiaohong $\mathrm{Zhu}^{1,2}$, Aili Wang ${ }^{1}$, Guiyan Wang ${ }^{1,3}$ and Yonghong Zhang ${ }^{1}$
}

To explore the co-effects of inflammation and endothelial dysfunction and insulin resistance (IR) on hypertension in a large Asian population. Data on demographic characteristics, blood pressure and other variables were collected; additionally, fasting plasma glucose, insulin and biomarkers, including C-reactive protein (CRP), soluble intercellular cell adhesion molecule-1 (sICAM-1), soluble E-selectin (sE-selectin) and angiotensin II were examined among 2553 Mongolian adults aged $\geqslant 20$ years. IR was assessed using the homeostasis model. The co-effects of elevated biomarkers of inflammation and endothelial dysfunction and IR on hypertension were analyzed. A total of 953 subjects were diagnosed with hypertension. Among hypertensive subjects, the levels of CRP (11.0 vs. $\left.6.7 \mathrm{mg} \mathrm{I}^{-1}\right)$, sICAM-1 (348.3 vs. $\left.335.9 \mathrm{ng} \mathrm{m}^{-1}\right)$, sE-selectin (20.9 vs. $\left.18.5 \mathrm{ng} \mathrm{ml}^{-1}\right)$ and angiotensin II $\left(61.3\right.$ vs. $\left.50.0 \mathrm{pg} \mathrm{m}^{-1}\right)$ were significantly higher among subjects with IR than those without IR; among normotensives, levels of CRP $\left(6.3\right.$ vs. $\left.5.2 \mathrm{mg} \mathrm{I}^{-1}\right)$ and sE-selectin $\left(20.1\right.$ vs. $\left.17.8 \mathrm{ng} \mathrm{ml}^{-1}\right)$ were higher among IR subjects than those without IR. The prevalence of hypertension was significantly higher among subjects with IR and 2 elevated biomarkers $(69.0 \%)$ and those with IR and $\geqslant 3$ elevated biomarkers $(79.3 \%)$ than among those with IR and no elevated biomarkers (45.9\%) and those with IR and 1 elevated biomarker (50.6\%). After adjusting for multivariate, the risk of hypertension was significantly associated with the coexistence of IR and any two or three elevated biomarkers (odds ratios (OR) $(95 \%$ confidence intervals $(\mathrm{Cls}))=2.55(1.60-4.06)$ and $3.19(1.15-8.86)$, respectively). In this Mongolian population, IR and elevated biomarkers of inflammation and endothelial dysfunction were related to hypertension and the coexistence of IR and elevated biomarkers of inflammation and endothelial dysfunction increased the risk of hypertension.

Hypertension Research (2012) 35, 513-517; doi:10.1038/hr.2011.229; published online 26 January 2012

Keywords: endothelial dysfunction; inflammation; insulin resistance

\section{INTRODUCTION}

Multiple cross-sectional studies ${ }^{1-4}$ and one cohort study ${ }^{5}$ have indicated that insulin resistance (IR) is associated with hypertension. Studies have also shown that inflammation and endothelial dysfunction are not only associated with atherosclerosis, cardiovascular disease and hypertension but also with IR in various settings. ${ }^{6-11}$ Inflammation and endothelial dysfunction may have an important role in the development of IR. ${ }^{12}$ Biomarkers of inflammation and endothelial dysfunction, such as circulating C-reactive protein (CRP), interleukin-6, soluble intercellular cell adhesion molecule-1 (sICAM-1), soluble vascular cell adhesion molecule-1 and soluble E-selectin (sE-selectin), have been reported to be associated with IR. ${ }^{13-15}$ In addition, studies have demonstrated that both endothelial dysfunction and IR are related to cardiovascular risk factors among hypertensive patients. ${ }^{16-18}$ However, few studies investigating the co-effects of inflammation and endothelial dysfunction and IR on hypertension in a large Asian population have been reported. The aim of this study was to investigate the co-effects of inflammation and endothelial dysfunction and IR on hypertension among a Mongolian population in Inner Mongolia, China.

\section{METHODS}

Study participants

From July 2002 to September 2003, a cross-sectional survey was conducted in 32 villages of two townships, Kezuohou Banner (county) and Naiman Banner, Inner Mongolia, China. These two adjacent townships are $100 \mathrm{~km}$ from Tongliao, a prefecture-level city in eastern Inner Mongolia, China. Most of the residents in the investigation field are Mongolian and have occupied the area for many generations. Of the 3475 Mongolians aged $\geqslant 20$ years sampled, 2589 provided informed consent and participated in the study. This study was approved by the Soochow University Ethics Committee.

${ }^{1}$ Department of Epidemiology, School of Public Health, Medical College of Soochow University, Suzhou, China; ${ }^{2}$ Department of Epidemiology, Center for Disease Prevention and Control in Suzhou Industrial Park District, Suzhou, China and ${ }^{3}$ Department of Health Care, Xiamen Maternal and Child Care Hospital, Xiamen, China

Correspondence: Dr Y Zhang, Department of Epidemiology, School of Public Health, Medical College of Soochow University, 199 Renai Road, Industrial Park District, Suzhou 215123, China.

E-mail: yhzhang@suda.edu.cn

Received 29 July 2011; revised 14 October 2011; accepted 2 November 2011; published online 26 January 2012 


\section{Data collection and examination}

Data on demographic characteristics, lifestyle risk factors, including cigarette smoking and alcohol drinking, as well as family and personal history of hypertension were collected using a standardized questionnaire administered by trained study staff. Cigarette smoking was defined as having smoked at least one cigarette per day for 1 year or more. Heavy alcohol consumption was defined as drinking at least $50 \mathrm{~g}$ of alcohol per day for 1 year or more.

Body weight and height were measured with subjects wearing light clothing and without shoes by trained staff. The body mass index was calculated as weight in kilograms divided by the square of height in meters. The waist circumference was measured at the level of $1 \mathrm{~cm}$ above the umbilicus. Overweight was defined as body mass index $\geqslant 25 \mathrm{~kg} \mathrm{~m}^{-2}$, and central obesity was defined as waist circumference $\geqslant 85 \mathrm{~cm}$ for males and $\geqslant 80 \mathrm{~cm}$ for females. ${ }^{19}$

Three blood pressure (BP) measurements were taken by four trained doctors using a standard mercury sphygmomanometer, and the mean of the three measurements was used in the analysis. Hypertension was defined as a systolic $\mathrm{BP} \geqslant 140$ and/or a diastolic BP $\geqslant 90 \mathrm{~mm} \mathrm{Hg}$ and/or use of antihypertensive medication within the previous 2 weeks.

Fasting blood samples were collected from all participants the morning after at least $8 \mathrm{~h}$ of fasting. Serum was subsequently isolated from the whole blood, and all serum samples were frozen at $-80^{\circ} \mathrm{C}$ until testing. Fasting plasma glucose was measured using a modified hexokinase enzymatic method. The concentrations of total cholesterol (TC), HDL-C (high-density lipoproteincholesterol) and triglycerides (TGs) were assessed enzymatically on a Beckman Synchrony CX5 Delta Clinical System (Beckman Coulter, Fullerton, CA, USA) using commercial reagents, and LDL-C (low-density lipoprotein-cholesterol) concentration was calculated by means of the Friedewald equation for participants who had $<4.52 \mathrm{mmoll}^{-1}\left(400 \mathrm{mg} \mathrm{dl}^{-1}\right)$ TG. ${ }^{20}$ High TG levels were defined as TG $\geqslant 1.70 \mathrm{mmoll}^{-1}$, high TC was defined as TC $\geqslant 5.18 \mathrm{mmoll}^{-1}$, high LDL-C was defined as LDL-C $\geqslant 3.37 \mathrm{mmoll}^{-1}$ and low HDL-C was defined as HDL-C $<1.04 \mathrm{mmoll}^{-1}$. CRP was determined with immunoturbidimetry on a Beckman Synchron CX5 Delta Clinical System using commercial reagents. sE-selectin and sICAM-1 were measured by an enzyme-linked immunosorbent assay (R\&D Systems, Minneapolis, MN, USA), which employs the quantitative sandwich enzyme immunoassay technique. ${ }^{21,22}$ Quantitative determination of immunoreactive angiotensin II was performed by a double antibody radioimmunoassay after reversed-phase sample extraction by means of phenylsilylsilica columns following the method previously reported by Emanuel et al. ${ }^{23}$ and a commercial radioimmunoassay kit.
The homeostasis model assessment method was used to calculate the insulin resistance index (HOMA-IR): HOMA-IR $=\left(\right.$ insulin $\left(\mathrm{mU} \mathrm{l}^{-1}\right) \times$ glucose $\left.\left(\mathrm{mmoll}^{-1}\right)\right) / 22.5,^{24}$ and IR was defined as HOMA-IR $>3.76 \mathrm{mU} \times \mathrm{mmoll}^{-2}$ (the fifth quintile). Elevated CRP, elevated sICAM-1, elevated sE-selectin and elevated angiotensin II were defined in this study sample as values of these markers that were greater than the top quintiles.

\section{Statistical analysis}

The mean values (standard deviations) or medians (quartile intervals) of continuous variables were calculated and compared using a Student's $t$-test or Wilcoxon rank test between subjects with IR and without IR across the hypertensive and normotensive groups. The prevalence and 95\% confidence intervals $(95 \% \mathrm{CI})$ for categorical variables were calculated, and $\chi^{2}$-tests were performed to compare between two groups.

The age and gender-adjusted prevalence of IR, high CRP, sICAM-1, sE-selectin and angiotensin II were computed using a direct standardized method and compared using weighted $\chi^{2}$-tests. The associations between hypertension and IR, biomarkers of inflammation and endothelial dysfunction were analyzed using a logistic regression model. Statistical analyses were conducted using SAS 9.1 statistical software (SAS Institute Inc., Cary, NC, USA), and all $P$-values were based on two-side tests with the significance level set to 0.05 .

\section{RESULTS}

Of the 2589 participants initially recruited, 2553 subjects were included in the analysis, and 36 subjects were excluded due to absence of fasting plasma glucose or insulin data. There were 953 hypertensive participants and 1600 normotensive participants in the sample of 2553 individuals.

Table 1 presents the comparison of characteristics between subjects with IR and without IR, grouped by BP status. In the hypertension group, subjects with IR were more likely to have higher prevalence of overweight, central obesity, high TG, high TC, and higher average levels of CRP, sICAM-1, sE-selectin and angiotensin II compared with those without IR. Similarly, among the normotensive group, the prevalence of overweight, central obesity and high TG, as well as the average levels of CRP and sE-selectin were significantly greater among subjects with IR than those without IR. In addition, hypertensive

Table 1 Characteristics of subjects grouped by both blood pressure and IR status

\begin{tabular}{|c|c|c|c|c|}
\hline \multirow[b]{2}{*}{ Variables } & \multicolumn{2}{|c|}{ Hypertension } & \multicolumn{2}{|c|}{ Normotension } \\
\hline & $I R(\mathrm{n}=234)$ & Non-IR $(\mathrm{n}=719)$ & $I R(\mathrm{n}=276)$ & Non-IR $(\mathrm{n}=1324)$ \\
\hline Age (age), $M\left(Q_{L}-Q_{U}\right)$ & $51(42-60)$ & $51(43-60)$ & $41(35-50)^{a}$ & $41(35-51)^{a}$ \\
\hline Male, \% (95\% Cl) & $51.3(44.8-57.7)$ & $49.7(46.0-53.3)$ & $35.5(29.8-41.2)^{a}$ & $35.6(33.1-38.2)^{a}$ \\
\hline Family history of hypertension, \% (95\% CI) & $23.1(17.6-28.5)$ & $25.3(22.1-28.5)$ & $8.0(4.7-11.2)^{a}$ & $5.7(4.4-6.9)^{a}$ \\
\hline Smoking, \% (95\% Cl) & $44.8(38.5-51.3)$ & $49.4(45.7-53.0)$ & 36.6 (30.9-42.3) & $43.4(40.8-46.1)$ \\
\hline Drinking, \% $(95 \% \mathrm{Cl})$ & $41.3(35.9-48.7)$ & $45.1(41.5-48.7)$ & $26.8(21.6-32.1)^{\mathrm{a}}$ & $27.0(24.6-29.4)^{a}$ \\
\hline Overweight, \% (95\% Cl) & $44.4(38.0-50.9)$ & $24.7(21.5-27.8)^{b}$ & $25.0(19.9-30.1)^{\mathrm{a}}$ & $12.5(10.8-14.3)^{a, b}$ \\
\hline Central obesity, \% (95\% Cl) & $64.1(57.9-70.3)$ & $50.2(46.5-53.9)^{b}$ & $48.2(42.3-54.1)^{a}$ & $31.4(28.9-33.9)^{a, b}$ \\
\hline High TG, \% (95\% Cl) & $34.8(28.6-40.9)$ & $23.1(20.0-26.2)^{b}$ & $22.5(17.6-27.5)^{a}$ & $10.3(8.7-12.0)^{a, b}$ \\
\hline High TC, \% $(95 \% \mathrm{Cl})$ & $21.5(16.1-26.8)$ & $13.0(10.6-15.5)^{b}$ & $8.7(5.4-12.1)^{a}$ & $7.0(5.6-8.4)^{a}$ \\
\hline High LDL-C, \% (95\% Cl) & $24.6(19.0-30.1)$ & $17.8(15.0-20.7)$ & $13.5(9.4-17.5)^{a}$ & $10.0(8.4-11.7)^{a}$ \\
\hline Low HDL-C, \% (95\% CI) & $47.8(44.4-54.3)$ & $35.5(32.0-39.1)^{b}$ & $37.1(31.3-42.8)^{a}$ & $36.3(33.7-38.9)$ \\
\hline $\operatorname{CRP}\left(\left.m g\right|^{-1}\right), M\left(Q_{L}-Q_{U}\right)$ & $11.0(5.2-18.8)$ & $6.7(4.4-13.3)^{b}$ & $6.3(4.1-12.1)^{\mathrm{a}}$ & $5.2(3.5-8.9)^{a, b}$ \\
\hline sICAM-1 (ng ml-1), $\bar{x} \pm s$ & $348.3 \pm 102.1$ & $335.9 \pm 97.7^{b}$ & $320.2 \pm 95.1^{a}$ & $322.9 \pm 96.9^{a}$ \\
\hline sE-selectin $\left(n g \mathrm{ml}^{-1}\right), M\left(Q_{\mathrm{L}}-\mathrm{Q}_{\mathrm{U}}\right)$ & $20.9(16.9-27.8)$ & $18.5(15.2-24.6)^{b}$ & $20.1(15.2-26.9)$ & $17.8(14.1-23.6)^{\mathrm{b}}$ \\
\hline Angiotensin II $\left(\mathrm{pg} \mathrm{ml}^{-1}\right), M\left(Q_{L}-Q_{U}\right)$ & $61.3(43.0-97.0)$ & $50.0(40.0-77.0)^{b}$ & $49.9(40.0-69.8)^{a}$ & $47.0(39.2-66.1)^{a}$ \\
\hline
\end{tabular}

Abbreviations: CRP, C-reactive protein; HDL-C, high density lipoprotein-cholesterol; IR, insulin resistance; LDL-C, low-density lipoprotein-cholesterol; M, median; $Q_{L}$, lower quartile; $Q_{U}$, upper quartile; sE-selectin, soluble sE-selectin; sICAM-1, soluble intercellular cell adhesion molecule-1; TC, total cholesterol; TG, triglycerides; $95 \% \mathrm{Cl}, 95 \%$ confidence interval. quartile; $\mathrm{sE}$-selectin, soluble $\mathrm{sE}$-selectin; $\mathrm{S}$ a

bNon-insulin resistance vs. insulin resistance, $P<0.05$. 
subjects were, regardless of IR or non-IR status, more likely to be older, male, have a family history of hypertension, report drinking, be overweight, have central obesity, high TG, high TC and low HDL-C as well as have higher average levels of CRP, sICAM-1 and angiotensin II compared with normotensive subjects.

The unadjusted and age-gender-adjusted prevalence of IR, elevated CRP, elevated sICAM-1, elevated sE-selectin, elevated angiotensin II and prevalence of coexistence of IR with elevated inflammatory and endothelial biomarkers are presented in Table 2. Both the unadjusted and age-gender-adjusted prevalence of IR, elevated CRP, elevated sICAM-1, elevated angiotensin II, prevalence of coexistence of IR with elevated CRP, elevated sICAM-1, elevated sE-selectin and elevated angiotensin II, but not sE-selectin, were higher among hypertensive subjects than among normotensive subjects. Furthermore, unadjusted and age-gender-adjusted prevalence of IR and coexistence of IR with 1,2 or $\geqslant 3$ elevated biomarkers of inflammation and endothelial dysfunction were all significantly greater among hypertensive participants than among normotensive participants.

The prevalence of hypertension in subjects without IR and without elevated biomarkers, among subjects with IR, and among subjects with the coexistence of IR and 1, 2 or $\geqslant 3$ elevated inflammatory and endothelial biomarkers are shown in Figure 1. The prevalence of hypertension among subjects without IR and without elevated biomarkers, with IR, with the coexistence of IR and 1 elevated biomarkers, coexistence of IR and 2 elevated biomarkers, coexistence of IR and $\geqslant 3$ elevated biomarkers were 29.9, 45.9, 50.6, 69.0 and 79.3\%, respectively. The prevalence of hypertension was significantly greater among subjects with IR than in those without IR and without elevated biomarkers; the prevalence of hypertension was significantly greater in subjects with IR and 1, 2 or $\geqslant 3$ elevated biomarkers than among those without IR and without elevated biomarkers. The prevalence of hypertension was significantly greater in subjects with IR and 2 or $\geqslant 3$ elevated biomarkers than in those with IR alone. However, there was no significant difference in the prevalence of hypertension between subjects with IR and $\geqslant 3$ elevated biomarkers and those with IR and 2 elevated biomarkers.

Normotensive participants served as the control in this study. Odds ratios (ORs) and 95\% CIs for hypertension associated with IR, elevated biomarkers of inflammation and endothelial dysfunction are presented in Table 3. Compared with those without IR and without elevated biomarkers, subjects with IR or elevated CRP, sICAM-1, sE-selectin or angiotensin II had a greater risk of hypertension before adjusting for age, gender and other factors. Furthermore, coexistence of IR with elevated CRP, elevated sICAM-1, elevated sE-selectin or elevated angiotensin II increased the risk of hypertension, especially when IR was accompanied with two or three elevated biomarkers. After adjusting for age, gender, family history of hypertension, smoking, drinking, overweight and high TG, the risk of hypertension associated with IR alone, elevated CRP alone, elevated sICAM-1 alone, elevated angiotensin II alone and with the coexistence of IR and elevated CRP or elevated angiotensin II remained significant. Moreover, the risk of hypertension associated with the coexistence of IR and any two or any three elevated biomarkers was highly significant; the ORs $(95 \% \mathrm{CI})$ were 2.55 (1.60-4.06) and 3.19 (1.158.86), respectively.

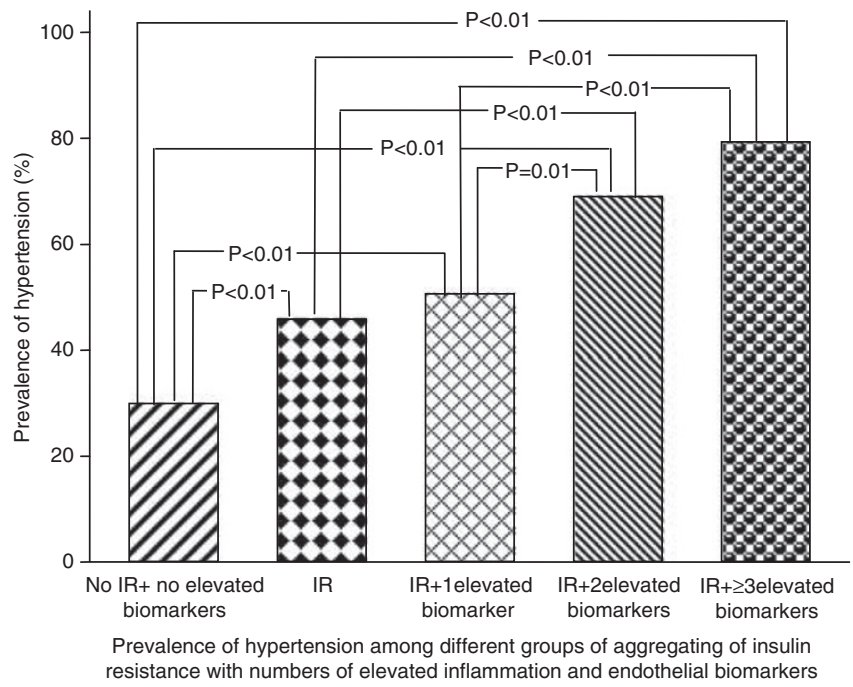

Figure 1 Prevalence of hypertension among different groups of aggregating of insulin resistance with numbers of elevated inflammation and endothelial biomarkers.

Table 2 Prevalence of IR and elevated inflammation and endothelial biomarkers in hypertensives and normotensives

\begin{tabular}{|c|c|c|c|c|}
\hline \multirow[b]{2}{*}{ Variables } & \multicolumn{2}{|c|}{ Hypertensives } & \multicolumn{2}{|c|}{ Normotensives } \\
\hline & Unadjusted & Age-gender adjusted & Unadjusted & Age-gender adjusted \\
\hline IR, \% & $24.5(21.8-27.3)$ & $26.3(23.1-29.5)$ & $17.3(15.4-19.1)^{a}$ & $17.0(15.2-18.8)^{\mathrm{b}}$ \\
\hline Elevated CRP, \% & $28.9(26.0-31.7)$ & $27.1(24.2-30.0)$ & $14.7(13.0-16.5)^{a}$ & $15.7(13.9-17.6)^{\mathrm{b}}$ \\
\hline Elevated sICAM-1, \% & $24.6(21.7-27.4)$ & $23.1(20.3-25.9)$ & $17.6(15.6-19.5)^{a}$ & $17.8(15.9-19.6)^{\mathrm{b}}$ \\
\hline Elevated sE-selectin, \% & $21.9(19.2-24.6)$ & $20.3(17.6-22.9)$ & $18.8(16.9-20.8)$ & $19.2(17.2-22.2)$ \\
\hline Elevated angiotensin II, \% & $25.7(23.0-28.5)$ & $25.5(22.8-28.4)$ & $16.0(14.2-17.8)^{a}$ & $15.9(14.2-17.6)^{\mathrm{b}}$ \\
\hline IR+elevated CRP, \% & $10.2(8.3-12.2)$ & $9.7(7.7-11.7)$ & $3.6(2.7-4.5)^{\mathrm{a}}$ & $3.7(2.8-4.7)^{\mathrm{b}}$ \\
\hline IR+elevated ICAM-1, \% & $7.2(5.5-8.9)$ & $7.1(5.3-8.9)$ & $3.3(2.4-4.2)^{\mathrm{a}}$ & $3.2(2.3-4.1)^{\mathrm{b}}$ \\
\hline IR+elevated E-selectin, \% & $7.2(5.5-8.9)$ & $7.0(5.2-8.8)$ & $4.2(3.2-5.2)^{\mathrm{a}}$ & $4.2(3.1-5.0)^{b}$ \\
\hline IR+elevated angiotensin II, \% & $8.2(6.4-9.9)$ & $7.8(5.9-9.7)$ & $2.9(2.1-3.7)^{\mathrm{a}}$ & $3.2(2.2-4.1)^{\mathrm{b}}$ \\
\hline IR+1 elevated biomarker, \% & $39.6(34.9-44.3)$ & $37.0(33.2-40.7)$ & $21.5(18.5-24.4)^{\mathrm{a}}$ & $21.3(18.6-23.9)^{\mathrm{b}}$ \\
\hline IR+2 elevated biomarkers, \% & $27.7(30.0-32.4)$ & $25.1(21.8-28.5)$ & $6.8(4.9-8.8)^{a}$ & $7.1(5.3-9.0)^{b}$ \\
\hline $\mathrm{IR}+\geqslant 3$ elevated biomarkers, \% & $8.2(5.0-11.5)$ & $6.1(3.8-8.3)$ & $1.0(0.2-1.8)^{a}$ & $0.9(0.2-1.6)^{b}$ \\
\hline
\end{tabular}

Abbreviations: CRP, C-reactive protein; IR, insulin resistance; sE-selectin, soluble sE-selectin; sICAM-1, soluble intercellular cell adhesion molecule-1.

${ }^{a}$ Compared with hypertensives for unadjusted prevalence, $P<0.05$.

${ }^{b}$ Compared with hypertensives for age-gender-adjusted prevalence, $P<0.05$. 
Table 3 ORs $(95 \% \mathrm{Cl})$ of hypertension associated with IR and elevated biomarkers of inflammation and endothelial dysfunction

\begin{tabular}{lcc}
\hline Variables & $\begin{array}{c}\text { Unadjusted OR } \\
(95 \% C I)\end{array}$ & $\begin{array}{c}\text { Multivariate-adjusted OR } \\
(95 \% C I)\end{array}$ \\
\hline Non-IR+low biomarkers & 1.00 & 1.00 \\
IR & $1.50(1.25-1.80)^{\mathrm{a}}$ & $1.25(1.00-1.58)^{\mathrm{a}}$ \\
Elevated CRP & $2.74(2.18-3.44)^{\mathrm{a}}$ & $1.55(1.17-2.04)^{\mathrm{a}}$ \\
Elevated ICAM-1 & $1.95(1.54-2.45)^{\mathrm{a}}$ & $1.35(1.02-1.78)^{\mathrm{a}}$ \\
Elevated E-selectin & $1.62(1.28-2.04)^{\mathrm{a}}$ & $1.09(0.83-1.44)$ \\
Elevated angiotensin II & $2.24(1.79-2.82)^{\mathrm{a}}$ & $1.76(1.34-2.30)^{\mathrm{a}}$ \\
IR+elevated CRP & $3.99(2.79-5.71)^{\mathrm{a}}$ & $1.81(1.16-2.84)^{\mathrm{a}}$ \\
IR+elevated ICAM-1 & $3.06(2.05-4.56)^{\mathrm{a}}$ & $1.55(0.97-2.56)$ \\
IR+elevated E-selectin & $2.38(1.63-3.47)^{\mathrm{a}}$ & $1.35(0.85-2.14)$ \\
IR+elevated angiotensin II & $3.97(2.68-5.88)^{\mathrm{a}}$ & $2.23(1.39-3.58)^{\mathrm{a}}$ \\
IR+1 elevated biomarker & $2.40(1.85-3.11)^{\mathrm{a}}$ & $1.37(1.00-1.88)^{\mathrm{a}}$ \\
IR+2 elevated biomarkers & $5.22(3.55-7.67)^{\mathrm{a}}$ & $2.55(1.60-4.06)^{\mathrm{a}}$ \\
IR+ $\geqslant 3$ elevated biomarkers & $8.98(3.62-22.33)^{\mathrm{a}}$ & $3.19(1.15-8.86)^{\mathrm{a}}$
\end{tabular}

Abbreviations: CRP: C-reactive protein; IR, insulin resistance; OR, odds ratio; sE-selectin: soluble sE-selectin; sICAM-1: soluble intercellular cell adhesion molecule-1; $95 \% \mathrm{CI}$, $95 \%$ confidence interval.

aOthers compared with non-IR+low biomarkers group, $P<0.05$

\section{DISCUSSION}

We examined the relationships between biomarkers of inflammation and endothelial dysfunction and IR, biomarkers of inflammation and endothelial dysfunction and hypertension, IR and hypertension, and the co-effect of IR and biomarkers of inflammation and endothelial dysfunction on hypertension in a large Mongolian population. This study included multiple biomarkers of inflammation and endothelial dysfunction, including CRP, sICAM-1, sE-selectin and angiotensin II; of the four, CRP, sICAM-1 and sE-selectin reflect the status of inflammation or the status of endothelial dysfunction. ${ }^{25-27}$ In addition to its role as a vasoconstrictor, angiotensin II induces endothelial dysfunction and inflammation, resulting in accelerated progression of atherosclerosis. ${ }^{28,29}$ Our study found that IR was related to the biomarkers of inflammation and endothelial dysfunction, both in subjects with hypertension and those without hypertension. Subjects with IR (HOMA-IR > 3.76) had higher CRP, sICAM-1, sE-selectin and angiotensin II levels than those without IR. Elevated biomarkers of inflammation and endothelial dysfunction were related to hypertension in subjects with and without IR. Hypertensive subjects had higher CRP, sICAM-1 and angiotensin II levels than normotensive subjects. The coexistence of IR and elevated biomarkers of inflammation and endothelial dysfunction increased the risk of hypertension, and the risk of hypertension for subjects with IR+2 elevated biomarkers or IR $+\geqslant 3$ elevated biomarkers was greater than for those with only IR or with any one elevated biomarker. These findings suggest that not only are biomarkers of inflammation and endothelial dysfunction alone and IR alone associated with hypertension, but their co-effect may also be associated with hypertension.

CRP, as a biomarker of systemic inflammation, has been reported to be a strong independent predictor of myocardial infarction, ischemic stroke, type 2 diabetes and hypertension. ${ }^{6,22}$ Moreover, experimental and epidemiological data have indicated that the inflammatory process is associated with impaired insulin sensitivity and the development of dysglycemic conditions. ${ }^{30}$ A study conducted in Peru among 1525 individuals $^{31}$ found that elevated CRP were significantly and positively associated with increased mean fasting insulin concentrations and mean HOMA-IR $(P<0.001)$ in both women and men. Women with CRP concentrations $>2.53 \mathrm{mgl}^{-1}$ (upper tertile) had a 2.18-fold increased risk of IR $(\mathrm{OR}=2.18$; 95\% CI 1.51-3.16) compared with those in the lowest tertile $\left(<0.81 \mathrm{mg} \mathrm{l}^{-1}\right)$. Among men, those in the upper tertile had a 2.54 -fold increased risk of IR $(\mathrm{OR}=2.54 ; 95 \%$ CI 1.54-4.20) compared with those in the lowest tertile. Another study $^{32}$ indicated that elevated sICAM-1 levels were independently associated with IR in a Taiwanese population. Chen et al. ${ }^{8}$ reported that the degree of IR was significantly correlated to concentrations of sE-selectin, sICAM-1 and soluble vascular cell adhesion molecule-1 among 28 healthy American volunteers. Ingelsson et al. ${ }^{33}$ found that vascular cell adhesion molecule-1, E-selectin and CRP were independently associated with metabolic syndrome (MetS) and increased HOMA-IR. Park et al. ${ }^{7}$ examined the cross-sectional and longitudinal relationships between CRP and IR and found a significant positive association between CRP and IR, in both cross-sectional and longitudinal results, even after adjusting for adiposity measurements and oxidative stress markers. Xu et al. ${ }^{34}$ found that CRP suppressed insulin signaling in endothelial cells. The studies by Park et al. ${ }^{7}$ and Xu et al. ${ }^{34}$ appear to support the hypothesis that inflammation and endothelial dysfunction introduce IR. Additional results indicate that IR may in turn exacerbate inflammation by increasing cytokine and adipochemokine expression (including TNF- $\alpha$, interleukin- 6 , leptin and others), elevating free fatty acid levels, and impairing endothelial nitric oxide synthase activity. ${ }^{35,36}$ Both inflammation and IR are two important links in the mechanism of hypertension; either systemic inflammation promotes the development of hypertension by introducing IR or IR promotes the development of hypertension by exacerbating inflammation. According to our findings that the coexistence of inflammation and IR increase the risk of hypertension, we presume that inflammation may partially and directly introduce hypertension by way of the inflammatory process in addition to IR; there is a co-effect between inflammation and IR on hypertension. Anuurad et al. ${ }^{37}$ conducted a study among African Americans and Caucasians and found that the synergistic relationship between inflammation and IR was a risk factor in coronary artery disease. Matsumoto et al. ${ }^{38}$ reported that among Japanese patients with type 2 diabetes mellitus and without a history of cardiovascular disease or stroke, the coexistence of IR and inflammation effectively predicted cardiovascular disease but not stroke.

In addition, results of multiple intervention studies indicated that lifestyle modification ${ }^{39,40}$ and pharmacologic treatment for hypertension, ${ }^{41,42}$ such as the angiotensin converting enzyme inhibitor, imidapril or the angiotensin II type 1 receptor antagonist, irbesartan, not only reduced BP values but also improved insulin sensitivity and endothelial function. Therefore, weight management or drug therapy may be necessary for hypertensive patients to reduce incidence of cardiovascular events.

There are limitations to the present study. First, it is a crosssectional study, and the co-effect of IR with elevated biomarkers of inflammation and endothelial dysfunction on hypertension should be elucidated in a cohort study. Second, $25 \%$ of the eligible individuals did not participate in the study because they were away from home or refused to respond, which may have caused selection bias. However, area inhabitants who did not participate in the study were likely unaware of their serum insulin, plasma glucose, biomarker levels and $\mathrm{BP}$ values, which may partially attenuate the selection bias.

This study has several advantages that should be mentioned. To our knowledge, this is the largest study to examine the association between hypertension and the co-effect of IR with elevated biomarkers of inflammation and endothelial dysfunction in a general Asian population. In addition, the study participants were homogeneous with respect to their genetic background and environmental exposures. 
The study data were collected with rigid quality control, and important co-variables were measured and controlled in the analysis.

In summary, in this Mongolian population, IR was associated with elevated biomarkers of inflammation and endothelial dysfunction; elevated biomarkers of inflammation and endothelial dysfunction were associated with hypertension, and the coexistence of IR and elevated biomarkers of inflammation and endothelial dysfunction increased the risk of hypertension.

\section{CONFLICT OF INTEREST}

The authors declare no conflict of interest.

\section{ACKNOWLEDGEMENTS}

We are deeply appreciative of the participants in the study and thank Kezuohou Banner Center for Disease Prevention and Control and Naiman Banner Center for Disease Prevention and Control for their support and assistance. Yonghong Zhang has received a grant (\#30972531) from the National Natural Science Foundation of China.

1 Lind $\mathrm{L}$, Berne $\mathrm{C}$, Lithell $\mathrm{H}$. Prevalence of insulin resistance in essential hypertension. J Hypertens 1995; 17: 1457-1462.

2 Ferrannini E, Buzzigoli G, Bonadonna R, Giorico MA, Oleggini M, Graziadei L, Pedrinelli $\mathrm{R}$, Brandi L, Bevilacqua S. Insulin resistance in essential hypertension. N Eng/ J Med 1987; 317: 350-357.

3 Saad MF, Rewers M, Selby J, Howard G, Jinagouda S, Fahmi S, Zaccaro D, Bergman RN, Savage PJ, Haffner SM. Insulin resistance and hypertension: the Insulin Resistance Atherosclerosis Study. Hypertension 2004; 43: 1-8.

4 Ferrari P, Weidmann P. Insulin, insulin sensitivity and hypertension. J Hypertens 1990; 8: 491-500.

5 Hirose H, Saito I, Kawabe H, Sarita T. Insulin resistance and hypertension: seven-year follow-up study in middle-aged Japanese men (the KEIO study). Hypertens Res 2003; 26: 795-800.

6 Anuurad E, Tracy RP, Pearson TA, Kim K, Berglund L. Synergistic role of inflammation and insulin resistance as coronary artery disease risk factors in African Americans and Caucasians. Atherosclerosis 2009; 205: 290-295.

7 Park K, Steffes M, Lee DH, Himes JH, Jacobs Jr DR. Association of inflammation with worsening HOMA-insulin resistance. Diabetologia 2009; 52: 2337-2344.

8 Chen NG, Holmes M, Reaven GM. Relationship between insulin resistance, soluble adhesion molecules, and mononuclear cell binding in healthy volunteers. J Clin Endocrinol Metab 1999. 84: 3485-3489.

9 Valle Jiménez M, Estepa RM, Camacho RM, Estrada RC, Luna FG, Guitarte FB. Endothelial dysfunction is related to insulin resistance and inammatory biomarker levels in obese prepubertal children. Eur J Endocrinol 2007; 156: 497-502.

10 Pinkney JH, Stehouwer CD, Coppack SW, Yudkin JS. Endothelial dysfunction: cause of the insulin resistance syndrome. Diabetes 1997; 46: S9-S13.

11 Perticone F, Sciacqua A, Scozzafava A, Ventura G, Laratta E, Pujia A, Federici M, Lauro R, Sesti G. Impaired endothelial function in never treated hypertensive subjects carrying the Arg972 polymorphism in the insulin receptor substrate-1 gene. J Clin Endocrinol Metab 2004; 89: 3606-3609.

12 Festa A, D'Agostino Jr R, Howard G, Mykkanen L, Tracy RP, Haffner SM. Chronic subclinical inflammation as part of the insulin resistance syndrome: the Insulin Resistance Atherosclerosis Study (IRAS). Circulation 2000; 102: 42-47.

13 Yudkin JS, Stehouwer CD, Emeis JJ, Coppack SW. C-reactive protein in healthy subjects: associations with obesity, insulin resistance, and endothelial dysfunction: a potential role for cytokines originating from adipose tissue? Arterioscler Thromb Vasc Biol 1999; 19: 972-978.

14 Leinonen E, Hurt-Camejo E, Wiklund O, Hulten LM, Hiukka A, Taskinen MR. Insulin resistance and adiposity correlate with acute-phase reaction and soluble cell adhesion molecules in type 2 diabetes. Atherosclerosis 2003; 166: 384-394.

15 Hossain M, Faruque MO, Kabir G, Hassan N, Sikdar D, Nahar Q, Ali L. Association of serum TNF-a and IL-6 with insulin secretion and insulin resistance in IFG and IGT subjects in a Bangladeshi population. Int J Diabetes Mellit 2010; 2: 165-168.

16 Perticone F, Maio R, Sciacqua A, Andreozzi E, lemma G, Perticone M, Zoccali C, Sesti G. Endothelial dysfunction and C-reactive protein are risk factors for diabetes in essential hypertension. Diabetes 2008; 57: 167-171.
17 Sheu WH, Jeng CY, Shieh SM, Fuh MM, Shen DD, Chen YD, Reaven GM. Insulin resistance and abnormal electrocardiograms in patients with high blood pressure. Am J Hypertens 1992; 5: 444-448.

18 Lima NKC, Abbasi F, Lamendola C, Reaven GM. Prevalence of insulin resistance and related risk factors for cardiovascular disease in patients with essential hypertension. Am J Hypertens 2009; 22: 106-111.

19 Cooperative Meta-analysis Group of China Obesity Task Force. Predictive values of body mass index and waist circumference to risk factors of related diseases in Chinese adult population. Chin J Epidemiol (in Chinese) 2002; 23: 5-10.

20 Friedewald WT, Levy RI, Fredrickson DS. Estimation of the concentration of low-density lipoprotein cholesterol in plasma, without use of the preparative ultracentrifuge. Clin Chem 1972; 18: 499-502.

21 Haber E, Koerner T, Page LB, Kliman B, Purnode A. Application of a radioimmunoassay for angiotensin I to the physiologic measurements of plasma renin activity in normal human subjects. J Clin Endocrinol Metab 1969; 29: 1349-1355.

22 Ridker PM. C-reactive protein and the prediction of cardiovascular events among those at intermediate risk: moving an inflammatory hypothesis toward consensus. J Am Coll Cardiol 2007; 49: 2129-2138.

23 Emanuel RL, Cain JP, Williams GH. Double antibody radioimmunoassay of renin activity and angiotensin II in human peripheral plasma. J Lab Clin Med 1973; 81: 632-640.

24 Matthews DR, Hosker JP, Rudenski AS, Naylor BA, Treacher DF, Turner R. Homeostatic model assessment: insulin resistance and $\beta$-cell function from fasting plasma glucose and insulin concentrations in man. Diabetologia 1985; 28: 412-419.

25 Zhang $\mathrm{C}$. The role of inflammatory cytokines in endothelial dysfunction. Basic Res Cardiol 2008; 103: 398-406.

26 Szmitko PE, Wang CH, Weisel RD, Almeida JR, Anderson TJ, Verma S. New markers of inflammation and endothelial cell activation, Part I. Circulation 2003; 108: 1917-1923.

27 Ridker PM, Hennekens CH, Buring JE, Rifal N. C-reactive protein and other markers of inflammation in the prediction of cardiovascular disease in women. $N$ Engl J Med 2000; 342: 836-843.

28 Schiffrin EL, Touyz RM. Multiple actions of angiotensin II in hypertension: benefits of AT1 receptor blockade. J Am Coll Cardiol 2003; 42: 911-913.

29 Pastore L, Tessitore A, Martinotti S, Toniato E, Alesse E, Bravi MC, Ferri C, Desideri G, Gulino A, Santucci A. Angiotensin II stimulates intercellular adhesion molecule-1 (ICAM-1) expression by human vascular endothelial cells and increases soluble ICAM-1 release in vivo. Circulation 1999; 100: 1646-1652.

30 Ndumele CE, Pradhan AD, Ridker PM. Interrelationships between inflammation, C-reactive protein, and insulin resistance. J Cardiometab Syndr 2006; 1: 190-196.

31 Gelaye B, Revilla L, Lopez T, Suarez L, Sanchez SE, Hevner K, Fitzpatrick AL, Williams MA. Association between insulin resistance and $\mathrm{C}$-reactive protein among Peruvian adults. Diabetol Metab Syndr 2010; 2: 30-35.

32 Hsua LA, Kob YL, Wu S, Teng MS, Chou HH, Chang CJ, Chang PY. Association of soluble intercellular adhesion molecule- 1 with insulin resistance and metabolic syndrome in Taiwanese. Metabolism 2009; 58: 983-988.

33 Ingelsson E, Hulthe J, Lind L. Inflammatory markers in relation to insulin resistance and the metabolic syndrome. Eur J Clin Invest 2008; 38: 502-509.

34 Xu JW, Morita I, Ikeda K, Miki T, Yamori Y. C-reactive protein suppresses insulin signaling in endothelial cells: role of spleen tyrosine kinase. Mol Endocrinol 2007. 21: $564-573$.

35 Shoelson SE, Lee J, Goldfine AB. Inflammation and insulin resistance. J Clin Invest 2006; 116: 1793-1801.

36 Vincent MA, Montagnani M, Quon MJ. Molecular and physiologic actions of insulin related to production of nitric oxide in vascular endothelium. Curr Diab Rep 2003; 3: $279-288$

37 Anuurad E, Tracy SP, Pearson TA, Kim K, Berglund L. Synergistic role of inflammation and insulin resistance as coronary artery disease risk factors in African Americans and Caucasians. Atherosclerosis 2009; 205: 290-295.

38 Matsumoto K, Fujita N, Ozaki M, Tominaga T, Ueki Y, Miyake S. Coexistence of insulin resistance and inflammation effectively predicts cardiac disease but not stroke in Japanese patients with type 2 diabetes mellitus. Diabetes Res Clin Pract 2006; 74: 316-321.

39 Fogari R, Zoppi A, Corradi L, Preti P, Mugellini A, Lazzari P, Derosa G. Effect of body weight loss and normalization on blood pressure in overweight non-obese patients with stage 1 hypertension. Hypertens Res 2010; 33: 236-242.

40 Hamdy O, Ledbury S, Mullooly C, Jarema C, Porter S, Ovalle K, Moussa A, Caselli A, Caballero AE, Economides PA, Veves A, Horton ES. Lifestyle modification improves endothelial function in obese subjects with the insulin resistance syndrome. Diabetes Care 2003; 26: 2119-2125.

41 Fogari R, Zoppi A, Salvadeo SA, Mugellini A, Lazzari P, Santoro T, Derosa G. Fibrinolysis and insulin sensitivity in imidapril and candesartan (FISIC study) recipients with hypertension. Hypertens Res 2011; 34: 509-515.

42 Derosa G, Salvadeo SAT. Endothelial function, blood pressure control, and risk modification: impact of irbesartan alone or in combination. Integr Blood Press Control 2010; 3: 21-30. 